\title{
Post Covid Neuropsychiatric Problems
}

\author{
Thapa $\mathbf{R}^{1}$, Subedi $\mathbf{S}^{2}$ \\ 1. Consultant Psychiatrist, Neuro-Cardio and Multispeciality Hospital, Biratnagar, Nepal 2. Professor, \\ Department of Psychiatry, UCMS, Bhairahawa, Nepal
}

E-mail *Corresponding author: thaparanjan2@gmail.com

\section{BACKGROUND}

The ongoing COVID-19 pandemic has already lead to at least 202 million documented infections and 4.3 million deaths worldwide. ${ }^{1}$ This pandemic has not only lead to acute morbidity and mortality but also persistent physical and neuropsychiatric sequelae among the people who recover from the acute infection. Persistent neuropsychiatric symptoms are common in critically ill patients discharged from an intensive care unit (ICU) ${ }^{2}$, but such problems are being reported from people who suffered only mild or even asymptomatic Covid-19 infection. Problems like cognitive impairment, delirium, extreme fatigue, and clinically relevant mood symptoms have been reported from patients who suffered mild or even asymptomatic infection. ${ }^{3,4}$

Recent evidence suggests that there is two way relationship between psychiatric illness and Covid-19. Psychiatric illness can be both a risk factor for and consequence of COVID-19. In a large electronic health record based cohort study of over 60,000 COVID-19 cases, a diagnosis of a psychiatric disorder during the last one year was associated with a $65 \%$ increased risk of COVID-19 when compared with a matched cohort of patients with physical health issues without psychiatric diagnoses. ${ }^{5}$ Furthermore, over the 3 months following COVID-19 diagnosis, $18 \%$ of patients were diagnosed with a psychiatric diagnosis, with nearly 6\% representing a new diagnosis (e.g., dementia, anxiety, and insomnia). Similar increases in incident psychiatric diagnoses among US adults with COVID-19 were reported by Czeisler et al. ${ }^{6}$ Etiologies of Neuropsychiatric Symptoms:

Covid-19 is caused by SARS CoV-2. Coronaviruses traditionally are considered pulmonary diseases, often with accompanying
GI symptoms. ${ }^{7}$ However, COVID-19 patients have demonstrated a high prevalence of neuropsychiatric symptoms. ${ }^{5}$ There are four proposed mechanisms of CNS involvement by SARSCoV-2.

First, neuropsychiatric symptoms of COVID-19 are often caused by the impact of delirium. Patients with severe covid-19 infection, especially the elderly are at high risk of delirium due to a combination of multiple factors like electrolyte abnormalities, liver inflammation, impaired renal function, hypoxia, hyperinflammation, and isolation.8,9 These patients who develop delirium frequently suffer from both acute and long-term neuropsychiatric effects. ${ }^{10,11}$

Second route by which SARS-CoV-2 can affect the CNS function is viral-induced immune reaction and autoimmunity. A viral induced inflammatory response can lead to blood brain barrier (BBB) dysfunction, resulting in immune cell infiltration and CNS tissue damage. ${ }^{8}$

Third route is SARS-CoV-2-induced coagulopathy. Viral invasion of vascular endothelium leading to activated thrombotic and inflammatory cascades can lead to cerebrovascular events. ${ }^{8}$ Stroke is the most common neurological finding on imaging of hospitalized patients for SARS-CoV-2. ${ }^{12}$. Furthermore, stroke itself is a risk factor for depression, and other neuropsychiatric problems.

Finally, direct viral invasion of the CNS, though uncommon, has been demonstrated. A few reports have identified the virus in the CNS, but this is rare, even among patients who are severely symptomatic. ${ }^{13,14}$

Neurocognitive Disorders:

There are few data regarding the long-term cognitive consequences of COVID-19. A study 
conducted among 279 patients hospitalized with COVID-19 found that 34\% reported memory loss and $28 \%$ described impaired concentration approximately 3 months after discharge..$^{15}$ In Taquet et al's large EHR study, new onset dementia following hospitalization for COVID19 was 2-3 times more common than what was observed after hospitalization for other medical events. $^{5}$

In cases of severe COVID-19, long-term cognitive deficits are likely the sequelae of delirium experienced during the acute phases of illness. Particularly in older patients, delirium is one of the most common symptoms in COVID19 patients presenting to the ED, and can be the only or primary symptom of SARS-CoV-2 infection. ${ }^{10}$ Delirium occurs in at least $30 \%$ of patients hospitalized with COVID-19 and is substantially more common in those requiring ICU admission. ${ }^{16,17,18}$. It is a known fact that delirium is a major risk for long term cognitive impairment. For example, a seminal study in adults with respiratory failure or shock admitted to the medical or surgical ICU identified global cognitive impairment (of equivalent severity to mild Alzheimer's disease) in $24 \%$ at 1 year after discharge. ${ }^{2}$ Delirium has also been described in COVID-19 patients who do not experience severe medical complications 16,17 , and there have been reports of "brain fog" among patients experiencing milder symptoms who were never hospitalized and presumably did not experience delirium ${ }^{3,4}$ Hence it is important that patients who are discharged after surviving covid-19 must be screened for neurocognitive effects of COVID19. Comorbidities that affect cognition (e.g., depression, anxiety, fatigue, sleep disturbance) and reversible causes (e.g., nutritional deficiencies, endocrinopathies, metabolic derangements) should be screened for and corrected as indicated. Patients with more pronounced symptoms may benefit from formal neuropsychological evaluation. There are no evidenced-based medications for neurocognitive problems due to COVID-19 (or similar conditions), but stimulants or medications used for the treatment of neurodegenerative disorders (e.g., acetylcholinesterase inhibitors, memantine) may be appropriate depending on patient comorbidities.

\section{Mood and Anxiety Disorders:}

Patients with COVID-19 infection are at increased risk for depression and anxiety both during the infection as well as after recovery from acute infection. ${ }^{19}$ At about 1 month after infection, $31-38 \%$ of patients report depressive symptoms, $22-42 \%$ report anxiety symptoms, and $20 \%$ report obsessive-compulsive symptoms. ${ }^{20,21}$ Rates vary depending on the population studied, methods used to evaluate symptoms, and how long after infection symptoms are assessed. ${ }^{19}$ One prospective study of patients in New York City described symptom severity during hospitalization and 14-17 days later. During hospitalization, 36\% of participants reported anxiety and 29\% reported depression; 14-17 days later, the prevalence of anxiety and depression had decreased $(9 \%$ and $20 \%$, respectively), but acute stress symptoms emerged in $25 \% .22$

While depressive and anxiety symptoms are more common than formal mood or anxiety diagnoses in COVID-19 survivors, a significant proportion of patients meet diagnostic criteria for a psychiatric disorder. In the study by Taquet et al., a new or recurrent anxiety disorder was documented in $12.8 \%$ and depressive disorder in 9.9\%. Severe COVID-19 disease was associated with higher rates of these diagnoses, but all patients with COVID-19 had higher rates of psychiatric diagnoses when compared to controls. ${ }^{5}$ Depressive and/or anxiety symptoms following COVID-19 are more likely in females and those with infected family members, post-infection physical discomfort, severe infection, elevated inflammatory markers, and prior psychiatric diagnoses. ${ }^{20,2123,24}$

Suicidal ideation is also elevated following COVID-19 diagnosis. In one study, $3.5 \%$ of respondents reported suicidality at 1 month following infection. ${ }^{20}$. There have been numerous case reports of COVID-19 patients attempting to kill themselves during or prior to hospitalization. ${ }^{25,26}$ However till date there is no evidence to suggest that the rate of suicide is any different among the patients recovered from Covid-19.

There have been few interventional studies targeting anxiety and depression in COVID-19 patients. Progressive muscle relaxation training and an internet-based integrated intervention 
(including self-help with relaxation, mindfulness, self-compassion skills) were both associated with lower depression and anxiety scale scores in patients admitted to Chinese hospitals.27,28,29 There are no well-defined pharmacologic guidelines for management of depression and anxiety following SARS-CoV-2 infection. ${ }^{30}$ However, the general approach to psychiatric symptom management in the medically ill can also be applied to this population. Medications must be chosen considering the general health of the patient and possible drug interaction. Selective serotonin reuptake inhibitors (SSRIs) are generally welltolerated options for depression and anxiety. However paroxetine may not be an appropriate choice due to its short half-life, anticholinergic side effects and increased risk of drug-drug interactions.

\section{Post-Traumatic Stress Disorder:}

The prevalence of PTSD among COVID-19 patients appears to range from 20 to $30 \%$, while the prevalence of less strictly defined posttraumatic stress symptoms (PTSS) varies widely. ${ }^{31,32,33,34}$ For example, in a study of more than 700 clinically stable, hospitalized COVID19 patients in China, $96.2 \%$ reported significant PTSS associated with COVID-19.32, where PTSS were observed in only $31 \%$ in a British cohort evaluated 4-8 weeks after discharge from a large university hospital. ${ }^{31}$ By comparison, in a study of 64 Korean COVID-19 patients interviewed approximately 2.5 months following hospital discharge, $20.3 \%$ met criteria for PTSD. ${ }^{33}$ And similarly, in a cohort of 185 Italian patients who had been hospitalized with COVID-19, 22.2\% met criteria for PTSD at a 2-week post-discharge follow-up visit. ${ }^{34}$ Younger age, female gender, need for ICU care and past history of psychiatric illness were identified as risk factors for development of PTSD/PTSS following SARSCoV-2 infection. ${ }^{31,34}$

Pharmacotherapy specific to PTSD/PTSS related to COVID-19 has not been studied. Basic principles of treatment for PTSD in the medically ill can be applied, considering potential drug-drug interactions and possible organ dysfunction when selecting medications and doses. Evidence supports the use of the serotonin-norepinephrine reuptake inhibitor (SNRI) venlafaxine and SSRIs ( other than
Paroxetine) for PTSD in medically ill patients. ${ }^{35}$ Exposure-based cognitive behavioral therapy (CBT) has the highest level of evidence in those with PTSD, while supportive counseling, resilience training and psychological first aid have some evidence in treating PTSD. ${ }^{36}$ Of note, psychological debriefing is not beneficial for PTSD, may cause harm, and should be avoided. ${ }^{36}$

\section{Psychotic Disorders:}

Some studies have suggested that rates of psychosis increase during epidemics and pandemics. An observational study from China reported a $25 \%$ increased incidence of psychotic disorders. ${ }^{39}$ This relationship has been largely attributed to the substantial psychosocial stress of the pandemic, but, as described previously, more direct mechanisms have also been suspected. Of interest, $0.9-4 \%$ of people exposed to viral infections develop psychosis ${ }^{38}$, which is much higher than the incidence in the general population of 15.2 in 100,000 persons. ${ }^{39}$. The ongoing CoroNerve surveillance study identified new-onset psychosis in 10 of the first 153 patients with acute COVID-19-related neuropsychiatric complications. ${ }^{17}$ There have been several cases from multiple countries describing first-episode psychotic symptoms in COVID-19 patients. ${ }^{40,41}$ COVID-19 psychosis is often characterized by notable disorganization and confusional features.40,41 Compared to patients who develop psychosis precipitated by pandemic-related stress, these patients may be less likely to suffer from paranoia or delusional content about COVID-19.40 They may also be less likely to have a family history of psychosis and more likely to present at an atypical age with subacute onset and relatively fast recovery following treatment with low-dose antipsychotics. ${ }^{40}$

It is important to know that medications used for treatment of Covid-19 can too lead to psychosis. Chloroquine and hydroxychloroquine, which were previously used in COVID-19 care, can cause hallucinations and other psychotic symptoms. High-dose corticosteroids, which are often used in treatment of severe COVID-19 infection, can cause psychotic symptoms. 


\section{CONCLUSION}

Recent evidences suggest that the impact of the COVID -19 extends far beyond acute morbidity and mortality. Many studies clearly show that the survivors of COVID-19 frequently suffer from a range of neuropsychiatric illness. These problems are seen not only in patients who suffered from severe acute illness but also among those who suffered from mild or asymptomatic acute illness. In this period of pandemic COVID-19 should be considered as a possible cause of psychosis, depression, anxiety or cognitive disorder. The general public as well as heath workers need to be educated about these sequelae of COVID-19. Physicians must be trained to detect and treat or refer cases suffering from the neuropsychiatric sequalae of covid-19. Comprehensive long term studies need to be carried out to fully understand the impact of this pathogen. Further studies are also needed to devise appropriate treatment plans to manage the neuropsychiatric sequelae of COVID-19.

\section{REFERENCES:}

1. Johns Hopkins University \& Medicine. COVID-19 Dashboard by the center for systems science and engeneering: https://coronavirus.jhu.edu/map.html

2. Pandharipande PP, Girard TD, Jackson JC, Morandi A, Thompson JL, Pun BT, et al. Long-term cognitive impairment after critical illness. $N$ Engl J Med Mass Medical Soc. 2013;369:1306-16.

3. Rubin R. As their numbers grow, COVID-19 "long haulers" stump experts. JAMA. 2020;324:1381-3.

4. Woo MS, Malsy J, Pöttgen J, Zai SS, Ufer F, Hadjilaou $A$, et al Frequent neurocognitive deficits after recovery from mild COVID-19. Brain Commun. 2020.

5. Taquet $M$, Luciano $S$, Geddes JR, Harrison PJ. Bidirectional associations between COVID-19 and psychiatric disorder: a study of 62,354 COVID-19 cases. Lancet Psychiatry. 2020;S2215-0366: 30462-4.

6. CzeislerMÉ, Lane RI, Petrosky E,Wiley JF, ChristensenA, Njai $R$, et al Mental health, substance use, and suicidal ideation during the COVID-19 pandemic United States, June 24-30, 2020. MMWR Morb MortalWkly Rep [Internet]. Centers for Disease Control and Prevention; 2020;69:1049-57. Available from: https://pubmed.ncbi.nlm.nih.gov/32790653.

7. Jasti $M$, Nalleballe K, Dandu V, Onteddu S. A review of pathophysiology and neuropsychiatric manifestations of COVID-19. J Neurol Springer. 2020;Jun;3(1):6.

8. Achar A, Ghosh C. COVID-19-associated neurological disorders: the potential route of CNS invasion and bloodbrain relevance. Cells. 2020;9:2360.

9. Oussalah A, Gleye S, Urmes IC, Laugel E, Barbé F, Orlowski $S$, et al. The spectrum of biochemical alterations associated with organ dysfunction and inflammatory status and their association with disease outcomes in severe COVID-19: a longitudinal cohort and

time-series design study. EClinicalMedicine. Elsevier. 2020;27: 100554.

10. Kennedy M, Helfand BKI, Gou RY, Gartaganis SL, Webb M, Moccia JM, et al. Delirium in older patients with COVID-19 presenting to the emergency department. JAMA Netw Open. 2020, 2020;(3):e2029540-0. https://doi.org/10.1001/jamanetworkopen. 2020.29540.

11. Helms J, Kremer S, Merdji H, Schenck M, Severac F, Clere-Jehl $R$, et al. Delirium and encephalopathy in severe COVID-19: a cohort analysis of ICU patients. Crit Care BioMed Central. 2020;24:1-11.

12. Jain $R$, Young $M$, Dogra S, Kennedy $H$, Nguyen $V$, Jones $S$, et al. COVID-19 related neuroimaging findings: a signal of thromboembolic complications and a strong prognostic marker of poor patient outcome. J Neurol Sci Elsevier. 2020;116923.

13. Moriguchi T, Harii N, Goto J, Harada D, Sugawara H, Takamino J, et al. A first case of meningitis/encephalitis associated with SARSCoronavirus-2. Int J Infect Dis Elsevier. 2020;94:55-8.

14. Al Saiegh F, Ghosh R, Leibold A, Avery MB, Schmidt $R F$, Theofanis $T$, et al. Status of SARS-CoV-2 in cerebrospinal fluid of patients with COVID-19 and stroke. J Neurol Neurosurg Psychiatry. 2020;91:846-8.

15. Garrigues E, Janvier P, Kherabi Y, Le Bot A, Hamon A, Gouze $H$, et al. Post-discharge persistent symptoms and health-related quality of life after hospitalization for COVID-19. J Inf Secur. 2020;81:e4-6.

16. Liotta EM, Batra A, Clark JR, Shlobin NA, Hoffman $S C$, Orban ZS, et al. Frequent neurologic manifestations and encephalopathyassociated morbidity in Covid-19 patients. Ann Clin Transl Neurol. 2020;7:2221-30.

17. Varatharaj A, Thomas N, Ellul MA, NWS D, Pollak TA, Tenorio EL, et al. Neurological and neuropsychiatric complications of COVID-19 in 153 patients: a UK-wide surveillance study. Lancet Psychiatry. 2020;7:875-82.

18. Helms J, Kremer S, Merdji H, Clere-Jehl R, Schenck M, Kummerlen $C$, et al. Neurologic features in severe SARSCoV-2 infection. N Engl J Med. 2020;382:2268-70.

19. Deng J, Zhou F, Hou W, Silver Z, Wong CY, Chang $O$, et al. The prevalence of depression, anxiety, and sleep disturbances in COVID-19 patients: a meta-analysis. Ann $N Y$ Acad Sci. Wiley-

Blackwell; 2020;Oct 2.:https://doi.org/10.1111/nyas.14506. 20. Mazza MG, De Lorenzo R, Conte C, Poletti S, Vai B, Bollettini I, et al. Anxiety and depression in COVID-19 survivors: role of inflammatory and clinical predictors. Brain Behav Immun Elsevier.

2020;89:594-600.

21. Cai X, Hu X, Ekumi IO, Wang J, An Y, Li Z, et al. Psychological distress and its correlates among COVID-19 survivors during early convalescence across age groups. Am J Geriatr Psychiatry Elsevier.2020;28:1030-9.

22. Parker C, Shalev D, Hsu I, Shenoy A, Cheung S, Nash $S$, et al. Depression, anxiety, and acute stress disorder 
among patients hospitalized with coronavirus disease 2019: a prospective cohort study. Psychosomatics Elsevier. 2020;S0033:3182-30262-0

23. Ma Y-F, Li W, Deng H-B, Wang L, Wang Y, Wang P$H$, et al. Prevalence of depression and its association with quality of life in clinically stable patients with COVID-19. J Affect Disord Elsevier.2020;275:145-8.

24. Nie X-D, Wang Q, Wang M-N, Zhao S, Liu L, Zhu Y$L$, et al Anxiety and depression and its correlates in patients with coronavirus disease 2019 inWuhan. Int J Psychiatry Clin Pract. Taylor EFrancis; 2020;1-6.

25. Epstein D, Andrawis W, Lipsky AM, Ziad HA, Matan M. Anxiety and suicidality in a hospitalized patient with COVID-19 infection. Eur J case reports Intern Med. 2020;7:001651.

26. Gillett G, Jordan I. Severe psychiatric disturbance and attempted suicide in a patient with COVID-19 and no psychiatric history. BMJ Case Reports CP. BMJ Specialist Journals. 2020;13:e239191.

27. Xiao C-X, Lin $Y-J$, Lin $R-Q$, Liu A-N, Zhong G-Q, Lan $C$-F. Effects of progressive muscle relaxation training on negative emotions and sleep quality in COVID-19 patients: a clinical observational study. Medicine (Baltimore). 2020;99:e23185.

28. Liu K, Chen Y, Wu D, Lin R, Wang Z, Pan L. Effects of progressive muscle relaxation on anxiety and sleep quality in patients with COVID-19. Complement Ther Clin Pract Elsevier. 2020;39101132.

29. Wei N, Huang B, Lu S, Hu J, Zhou X, Hu C, et al. Efficacy of internet-based integrated intervention on depression and anxiety symptoms in patients with COVID-19. J Zhejiang Univ Sci B Springer. 2020;21:4004.

30. Khawam E, Khouli H, Pozuelo L. Treating acute anxiety in patients with COVID-19. Cleve Clin I Med. 2020;May 14:doi:https://doi.org/10.3929/ccjm.87a.ccc016.

31. Halpin SJ, McIvor C, Whyatt G, Adams A, Harvey O, McLean L, et al Postdischarge symptoms and rehabilitation needs in survivors of COVID-19 infection: a crosssectional evaluation. J Med Virol. 2020;Jul 30:doi:https://doi.org/10.1002/jmv.26368.

32. Bo HX, Li W, Yang Y, Wang Y, Zhang Q, Cheung T, et al Posttraumatic stress symptoms and attitude toward crisis mental health services among clinically stable patients with COVID-19

in China. Psychol Med. Cambridge University Press; 2020;Mar $27:$ doi:https://doi.org/10.1017/S0033291720000999.

33. ChangMC, Park D. Incidence of post-traumatic stress disorder after coronavirus disease. Healthcare (Basel). 2020;8:373.

34. Yang Y, Li W, Zhang Q, Zhang L, Cheung T, Xiang YT. Mental health services for older adults in China during the COVID-19 outbreak. Lancet Psychiatry. 2020;7:e19.

35. Ravindran LN, Stein MB. Pharmacotherapy of PTSD: premises, principles, and priorities. Brain Res. 2009;1293:24-39.

36. Freedman SA. Psychological interventions to prevent PTSD. Psychiatr Ann. 2019;49:314-9.
37. HuW, Su L, Qiao I, Zhu J, Zhou Y. COVID-19 outbreak increased risk of schizophrenia in aged adults. PsychChinaXiv. 2020;10.202003.00003.

38. Brown E, Gray R, Lo Monaco S, O'Donoghue B, Nelson B, Thompson $A$, et al. The potential impact of COVID-19 on psychosis: a rapid review of contemporary epidemic and pandemic research. Schizophr Res. 2020;222:79-87.

39. McGrath J, Saha S,Welham J, El Saadi O,MacCauley C, Chant D. A systematic review of the incidence of schizophrenia: thedistribution of rates and the influence of sex, urbanicity, migrant

status and methodology. BMC Med. 2004;2:13.

40. Parra A, Juanes A, Losada CP, Álvarez-Sesmero S, Santana VD, Martí I, et al. Psychotic symptoms in COVID-19 patients. A retrospective descriptive study. Psychiatry Res. 2020;291:113254.

41. Tariku M, Hajure M. Available evidence and ongoing hypothesis on corona virus (COVID-19) and psychosis: is corona virus and psychosis related? A narrative review. Psychol Res Behav Manag. 2020;13:701-4. 